\title{
Multi-agent-based bus route optimisation for restricting passenger traffic bottlenecks in disaster situations
}

\author{
Sayaka Morimoto* \\ The University of Electro-Communications, \\ 1-5-1 Chofugaoka, Chofu-shi, \\ Tokyo 182-8585, Japan \\ Email: sayaka@cas.hc.uec.ac.jp \\ *Corresponding author
}

\section{Takahiro Jinba}

Mizuho Information \& Research Institute,

2-3 Kanda-Nishikicho, Chiyoda-ku,

Tokyo 101-8443 Japan

Email: takahiro412.c@gmail.com

\section{Hiroto Kitagawa}

TIS Inc.,

8-17-1, Nishishinjuku,

Shinjuku-ku, Tokyo 160-0023, Japan

Email: kitagawahiroto0829@gmail.com

\section{Keiki Takadama}

The University of Electro-Communications, 1-5-1 Chofugaoka, Chofu-shi,

Tokyo 182-8585, Japan

Email: keiki@inf.uec.ac.jp

\section{Takahiro Majima}

National Maritime Research Institute, 6-38-1, Shinkawa, Mitaka-shi,

Tokyo 181-0004, Japan

Email: majy@nmri.go.jp

\section{Daisuke Watanabe}

Tokyo University of Marine Science and Technology, 4-5-7, Konan, Minato-ku, Tokyo 108-8477, Japan

Email: daisuke@kaiyodai.ac.jp 


\title{
Mitujiro Katuhara
}

\author{
Socio Tech Data, \\ 1-5-1, Hanekami, Hamura-shi, \\ Tokyo 205-0016, Japan \\ Email: kat-151@mail.bbexcite.jp
}

\begin{abstract}
This paper focuses on the passenger traffic bottlenecks occurring in the bus route network in disaster situations and proposes the multi-agent-based bus route network optimisation method to resolve such bottlenecks by generating the networks which can effectively transport many stranded persons who wait around the station as the bottlenecks. For this purpose, the proposed method modifies the bus route networks generated as usual situations to suitably generate the bus lines and redistribute the buses among the lines according to the number of passengers. The intensive simulations have revealed the following implications: the proposed method 1) can optimise the bus route network which is suitable for the bottlenecks; 2) optimises the bus route network which can cope with the hard disaster situations without an additional buses; 3) has the capability of decreasing a risk of the bottlenecks by concentrating on a modification of the lines having the bottleneck stations.
\end{abstract}

Keywords: route optimisation; multi-agent system; traffic bottleneck; disaster; stranded persons.

Reference to this paper should be made as follows: Morimoto, S., Jinba, T., Kitagawa, H., Takadama, K., Majima, T., Watanabe, D. and Katuhara, M. (2016) 'Multi-agent-based bus route optimisation for restricting passenger traffic bottlenecks in disaster situations', Int. J. Automation and Logistics, Vol. 2, Nos. 1/2, pp.153-177.

Biographical notes: Sayaka Morimoto received her BE degree from The University of Electro-Communications, Japan in 2014. She is currently a Master student in The University of Electro-Communications. Her research interests include the transportation in disaster, multi-agent system and the prevention of natural disasters.

Takahiro Jinba received his BE degree in 2013 and ME degree in 2015, both from The University of Electro-Communications, Japan. He joined Mizuho Information \& Research Institute from 2015. His research interests include flights optimisation, evolutionary algorithm and fleet assignment problem.

Hiroto Kitagawa received his BE degree in 2012 and ME degree in 2014, both from The University of Electro-Communications, Japan. He joined TIS Inc. in 2014. His research interests include the transportation in disaster, complex networks, clustering and genetic algorithm.

Keiki Takadama received his Doctor of Engineering degree from The University of Tokyo, Japan in 1998. He joined Advanced Telecommunications Research Institute (ATR) International from 1998 to 2002 as the Visiting Researcher and worked at Tokyo Institute of Technology from 2002 to 2006 as a Lecturer. He moved to The University of Electro-Communications as Associate Professor in 2006 and is currently a Professor since 2011. His research interests include multi-agent system, distributed artificial intelligence, autonomous system, and emergent computation. 
Takahiro Majima joined National Maritime Research Institute in 1992, where he is currently the head of the logistics research group. He received his Doctor of Engineering degree from Tokyo Institute of Technology, Japan, in 2009. His research interests include complex network, logistics and application of multi-agent system.

Daisuke Watanabe received his Doctor of Engineering degree in 2006 from The University of Tsukuba. He transferred from National Maritime Research Institute to Tokyo University of Marine Science and Technology in 2008. His primary research field is networks and logistics.

Mitujiro Katuhara received his Doctor of Engineering degree in 1972 from The University of Tokyo, Japan. He worked for NMRI from 1973 to 2006 . He is currently a President of SocioTechData. He was interested in the environmental issues, the safety of industries, the transportation economics, and the prevention of natural disasters.

This paper is a revised and expanded version of a paper entitled 'Multi-agent based bus route optimization for restricting passenger traffic bottlenecks in disaster situations' presented at The 18th Asia Pacific Symposium on Intelligent and Evolutionary Systems, Singapore, 10-12 November 2014.

\section{Introduction}

When disaster occurs, a large number of persons cannot return to their home due to the suspended most public transportations (Central Disaster Management Council, 2005; Cabinet Office and the Central Disaster Prevention Council, 2011). In such a situation, a bus service has the one of candidates to transport many stranded persons instead of the other transportations (e.g., the rail transport). For the route network optimisation in an ordinary situation (i.e., the situation without disaster), a lot of methods have been proposed. For example, the heuristics-based optimisation methods were proposed by Baaj and Mahmassani (1995) and Zhao and Ubaka (2004), while the meta-heuristic-based optimisation methods with the simulated annealing were proposed by Fan and Machemehl (2006) and Zhao and Zeng (2007) and those with genetic algorithm were proposed by Chakroborty and Wivedi (2002), Gen et al. (2008) and Ngamchai and Lovell (2003). In comparison with these methods, we apply a multi-agent-based route network optimisation method which considers each route as an agent to generate the route network for the disaster situation. This is because it is easy to deal with adding/deleting the routes or modifying their routes according to the dynamic condition change caused by the disaster. In this point of view, Majima et al. (2015) proposed the multi-agent-based route optimisation method, and succeeded to minimise both the costs of passengers (i.e., the total travelling time) and bus companies (i.e., the number of buses). Our previous research extended Majima's method for the damage situation caused by the destruction of roads to minimise the damage of the road destruction (Kitagawa et al., 2014). However, these studies cannot directly cope with the problem of a bus service called passenger traffic bottlenecks caused in the disaster situation, i.e., the number of passengers exceeds usual demands which are accumulated by the number of stranded persons around bus stops. The issue of the passenger traffic bottlenecks is very significant because it is necessary not to cause such bottlenecks to prevent secondary disasters such as the traffic 
accident and congestion. In addition to this problem, the studies Kitagawa et al. (2014) and Majima et al. (2015) have no strict restrictions of the number of buses meaning that the required buses can be provided to transport all passengers, which is impractical in the disaster situations.

To tackle the above problem, i.e., the passenger traffic bottlenecks, this paper proposes a new multi-agent-based bus route network optimisation method which can generate the networks for effectively transporting many stranded persons including ones who wait around the station as the passenger traffic bottlenecks. For this purpose, the proposed method focuses on both the bus lines and the number of buses around the bottleneck stations by modifying the bus route network generated as usual situations. To investigate the effectiveness of the proposed method, this paper compares our methods with the conventional one through the benchmark problem of the bus route network optimisation (Mandl, 1979). In particular, this paper is the extended version of our paper (Morimoto et al., 2014) presented at IES2014 conference for the special issue by including the detailed analysis of a hard disaster situation and by adding the new experiment for the comprehensive disaster situation from slight to extreme case.

This paper is organised as follows. Section 2 introduces the route network optimisation method through a comparison the conventional method with the proposed one. The urban transport bench mark problem is described in Section 3. The three kinds of experiments are conducted and their results are discussed in Section 4. Finally, our conclusions are given in Section 5.

\section{Route network optimisation method based on multi-agent system for disaster situations}

\subsection{Bus route network optimisation problem}

In general, the bus route network optimisation problem (Mandl, 1979) includes the map with positions of the bus stops (referred as the stations) and the roads, and an origin-destination (OD) table which indicates the number of passengers between stations. This problem assumes that the capacity of a bus and the speed of a bus are fixed. A line is expressed as a set of the stations sorted at the stopping order, and the bus stops at the stations one by one and runs back and forth.

As the evaluation of the bus route network, the evaluation value $Z$ of the route network is calculated by

$$
Z=\sum_{S_{i} \neq S_{j}} T_{S_{i}, S_{j}} D_{S_{i}, S_{j}}+\omega_{1} \sum_{L_{k}} B_{L_{k}}
$$

where $T_{S_{i} . S_{j}}$ represents the travelling time of the passengers from the origin station $S_{i}$ to the destination station $S_{j}, D_{S_{i}, S_{j}}$ represents the demand of the passengers which occur per unit time from $S_{i}$ to $S_{j}, B_{L_{k}}$ represents the number of buses in the line $L_{k}$ and $\omega_{1}$ indicates the weighting coefficient value. The lower the evaluation value $Z$ shows, the more superior the route network is, i.e., the good route network can transport the passengers with a short time and/or a small number of buses. In this paper, this estimation value $Z$ is only used to generate the primary route network and set the number of buses that each line has. 


\subsection{Algorithm}

As described in Section 1, the conventional route network optimisation method based on the multi-agent system MAS (such as the Majima's method) cannot directly cope with the passenger traffic bottlenecks which are often caused in disaster situations. To tackle this issue, we extend the Majima's method to concentrate on both the bus lines and the number of buses around the bottleneck stations by changing the following two parts:

1 the passengers at the bottleneck station are weighted (see Section 2.3)

2 the line having enough buses gives them to the other line having deficient buses (see Section 2.4).

Note that the algorithm of both methods has no random parameters, which means that the optimised route network is always same (i.e. both Majima's and proposed method are deterministic).

\subsubsection{Basic algorithm (by the Majima's method)}

Before explaining the proposed method, this subsection starts to explain the Majima's method as the conventional route network optimisation method (Majima et al., 2015). Its flowchart is shown in Figure 1. First, the primary route network which consists of many lines and covers all of stations is loaded (\#1-1) or generated (\#1-2). Second, the method calculates the route that passengers take to travel home according to OD using Dijkstra's algorithm (\#2). Third, some unused lines are deleted if they exist (\#3). Fourth, for each line this method estimates the evaluation value $P$ based on the number of passengers and buses, and sorts the lines in the descending order according to $P(\# 4)$ for preparation of evolving lines. Fifth, $k$, the number of the target line, is set to 0 (\#5). Sixth, this method attempts to evolve the $k^{\text {th }}$ line having the highest $P$ to maximise its own evaluation value $P$ (\#6-1) by either adding or removing one station. When the line can be evolved (\#6-1 YES), this method returns to calculate the route of the passengers (\#2). When the line cannot be evolved (\#6-1 NO), on the other hand, $k$ is incremented until $k$ reaches the number of lines (\#6-2 NO) and this method returns to attempt to evolve the $k^{\text {th }}$ line (\#6-1). Finally, when $k$ exceeds the number of lines (i.e., all lines cannot increase their own evaluation value $P$ through the evolution), the optimisation is terminated.

\subsubsection{Improved algorithm (by the proposed method)}

The flowchart of the proposed method is shown in Figure 2. The difference between the Majima's method and our method is emphasised by the bold fonts (i.e., the mechanisms $\# 3$, \#4, and \#6-1) and the thick bordered box which indicates the redistribution of buses (\#3.5). Regarding these differences, our method improve deleting the unused line more effectively to cope with the disaster situation in the mechanisms \#3 (see Section 2.4), estimating the evaluation value $P$ of the line to cope with the bottleneck situation in the mechanisms \#4 (see Section 2.3.1), estimating how much $P$ will increase through the line evolution in the mechanisms \#6-1 (see Section 2.3.2), and redistributing the buses between the lines to relieve the passengers congestion the lines in the mechanisms \#3.5 (see Section 2.4). 
Figure 1 Flowchart of the conventional route optimisation method

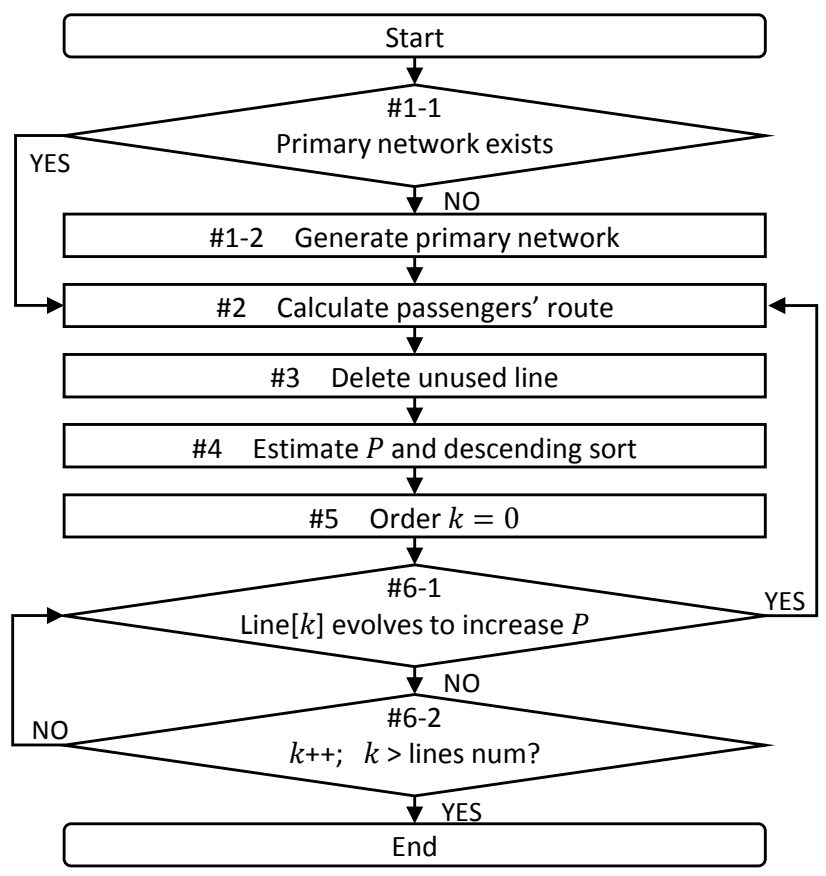

Source: Majima et al. (2015)

Figure 2 Flowchart of the proposed route optimisation method

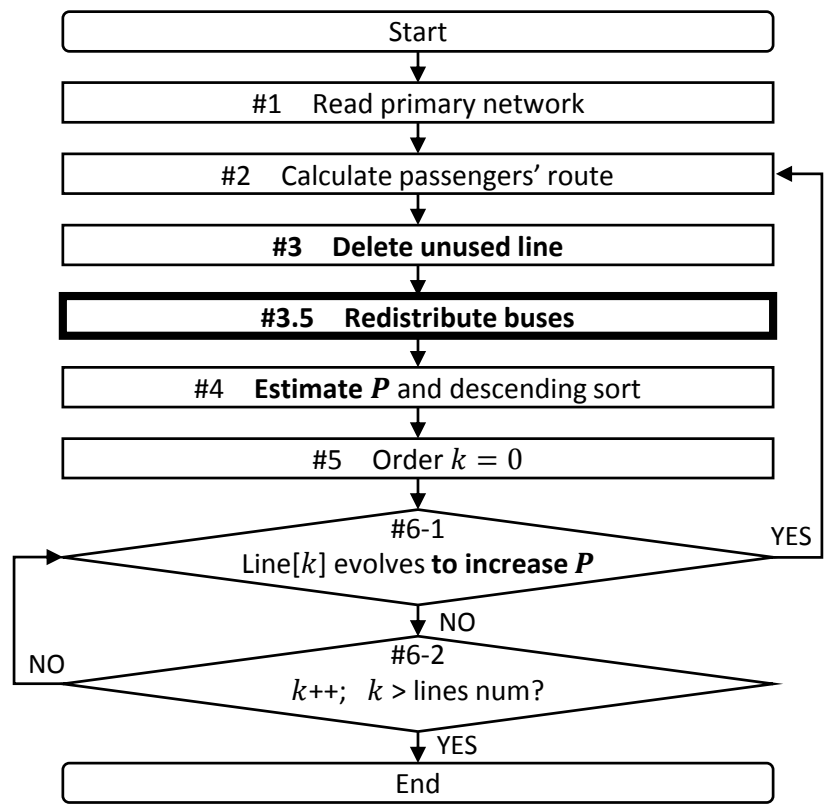




\subsection{Detailed mechanisms}

This subsection describes the detailed mechanisms of the algorithm explained in Section 2.2.

\subsubsection{Selection of line (\#4 estimate P and descending sort)}

\subsubsection{Basic algorithm (by the Majima's method)}

As the fundamental evaluation of the bus lines (not the evaluation of the whole bus network), the evaluation value $P$ employed to decide the target line for its route evolution is calculated by

$$
P_{L_{k}}^{\text {step }}=R_{L_{k}}^{\text {step }}-\omega_{2} B_{L_{k}}^{\text {step }}
$$

where step represents the number of evolution steps, $R_{L_{k}}^{\text {step }}$ represents the number of passengers in the line $L_{k}$ and $\omega_{2}$ indicates the weighting coefficient value. The higher evaluation value $P$ shows, the more superior line is, i.e., the good line can transport many passengers with a small number of buses. After calculating all evaluation value $P$ of the lines, all lines are sorted in the descending order according to $P$ to select the line having a high evaluation value to evolve it.

\subsubsection{Improved algorithm (by the proposed method)}

To cope with the bottleneck situation by modifying the lines including the bottleneck stations, equation (2) is modified to the following equation (3).

$$
P_{L_{k}}^{\text {step }}=\alpha R_{B}^{\text {step }}+\beta R_{N}^{\text {step }}-\omega_{2} B_{L_{k}}^{\text {step }}
$$

where $R_{B}^{\text {step }}$ represents the number of passengers in the bottleneck stations of the line $L_{k}$, $R_{N}^{\text {step }}$ represents one in the other stations (i.e., the non-bottleneck stations), $\alpha(\alpha>1)$ and $\beta(\beta<1)$ indicates the weighting coefficient value. By setting a high value $\alpha$, the value $P_{L_{k}}^{\text {step }}$ of the line which contains the bottleneck stations becomes higher than the one in the Majima's method shown in equation (2). Note that our method weights the passengers not only who pile up around the station but also who can return to their destinations as the served passengers.

\subsubsection{Evolution of line (\#6.1 line [k] evolves to increase P)}

As the fundamental of the line evolution, both methods evolve the line having the highest evaluation value $P$ by either

1 adding an adjacent station

2 removing a station belonging to the line.

The line estimates how the evaluation value $P$ will increase after the line evolution, and selects either an adding-typed evolution or a removing-typed evolution which can increase the most of the evaluation value $P$. 
Figure 3 shows an image of adding or removing a station where the circle represents a station, the thin line represents a road and the thick line represents a bus line passing over the road. In case of adding a station [Figure 3(a)], the adjacent stations D and E are added as the target stations. In this example, the line is evolved to add the station E. Note that a line can be evolved to add any station not only in the end of the line but also in its middle. In case of removing a station [Figure 3(b)], on the other hand, the stations A, B and $\mathrm{E}$ are removed as the target stations. In this example, the line is evolved to remove the station B. When the station in the middle of the line is removed, the stations in both sides are connected.

Figure 3 Line evolution, (a) adding (b) removing

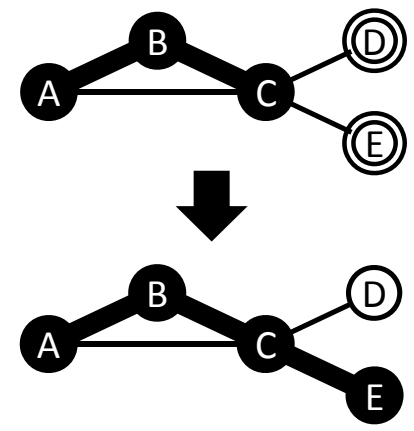

(a)

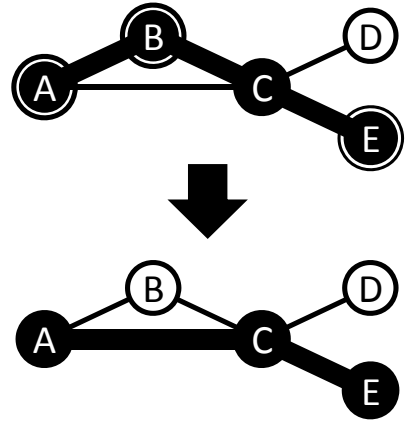

(b)

\subsubsection{Basic algorithm (by the Majima's method)}

In the conventional method, an increased difference $A$ of the evaluation value $P$ is calculated by

$$
A^{\text {step }}=\left(R_{L_{k}}^{\text {step }+1}-R_{L_{k}}^{\text {step }}\right)-\omega_{2}\left(B_{L_{k}}^{\text {step }+1}-B_{L_{k}}^{\text {step }}\right)
$$

where $R_{L_{k}}^{\text {step+1 }}$ and $B_{L_{k}}^{\text {step }+1}$ are the estimated values of the number of passengers and buses after the line evolution. The value $A$ becomes large/small when the number of passengers increases/decreases and/or the number of buses decreases/increases through the line evolution. The line compares all types of the evolutions from the viewpoint of $A$ and selects the one type which has the highest $A$. When all $A$ is less than 0 , the method evolves the next line. When all of the lines cannot increase their $P$, the route network optimisation is terminated.

\subsubsection{Improved algorithm (by the proposed method)}

To cope with the bottleneck situation, the change of equation (4) to equations (5), (6), and (7) promote to select the line having the bottleneck stations for its evolution.

$$
A^{\text {step }}=\left(R_{L_{k}}^{\text {step }+1}-R_{L_{k}}^{\text {step }}\right)-\omega_{3} R_{L_{k}}^{\text {step }}\left(T_{L_{k}}^{\text {step }+1}-T_{L_{k}}^{\text {step }}\right)
$$




$$
\left(R_{L_{k}}^{\text {step }+1}-R_{L_{k}}^{\text {step }}\right)= \begin{cases}\alpha\left(R_{L_{k}}^{\text {step }+1}-R_{L_{k}}^{\text {step }}\right), & \text { if bottleneck } \\ \beta\left(R_{L_{k}}^{\text {step }+1}-R_{L_{k}}^{\text {step }}\right), & \text { else }\end{cases}
$$

where $T_{L_{k}}^{\text {step }}$ represents the waiting time of passengers in the line $L_{k}$. Since it is almost impossible to add some buses from the outside in the disaster situations, the total number of buses is fixed in the route network. Based on this assumption, our method removes the term $\left(B_{L_{k}}^{\text {step }+1}-B_{L_{k}}^{\text {step }}\right)$ which is not changed while adds the term $\left(T_{L_{k}}^{\text {step }+1}-T_{L_{k}}^{\text {step }}\right)$ to decreases the waiting time of the lines. Furthermore, the term $\left(R_{L_{k}}^{\text {step }+1}-R_{L_{k}}^{\text {step }}\right)$ of equation (5) is also modified according to the following two cases to transfer passengers around the bottleneck stations on a preferential basis:

$1 \alpha(>1)$ is multiplied to the term $\left(R_{L_{k}}^{\text {step }+1}-R_{L_{k}}^{\text {step }}\right)$ in the bottleneck stations as shown in equation (6)

$2 \beta(<1)$ is multiplied to the term $\left(R_{L_{k}}^{\text {step }+1}-R_{L_{k}}^{\text {step }}\right)$ in the non-bottleneck stations as shown in equation (7).

This contributes to transporting more passengers in the bottleneck stations than those in the non-bottleneck stations.

Figure 4 Image of adding the station by the proposed method
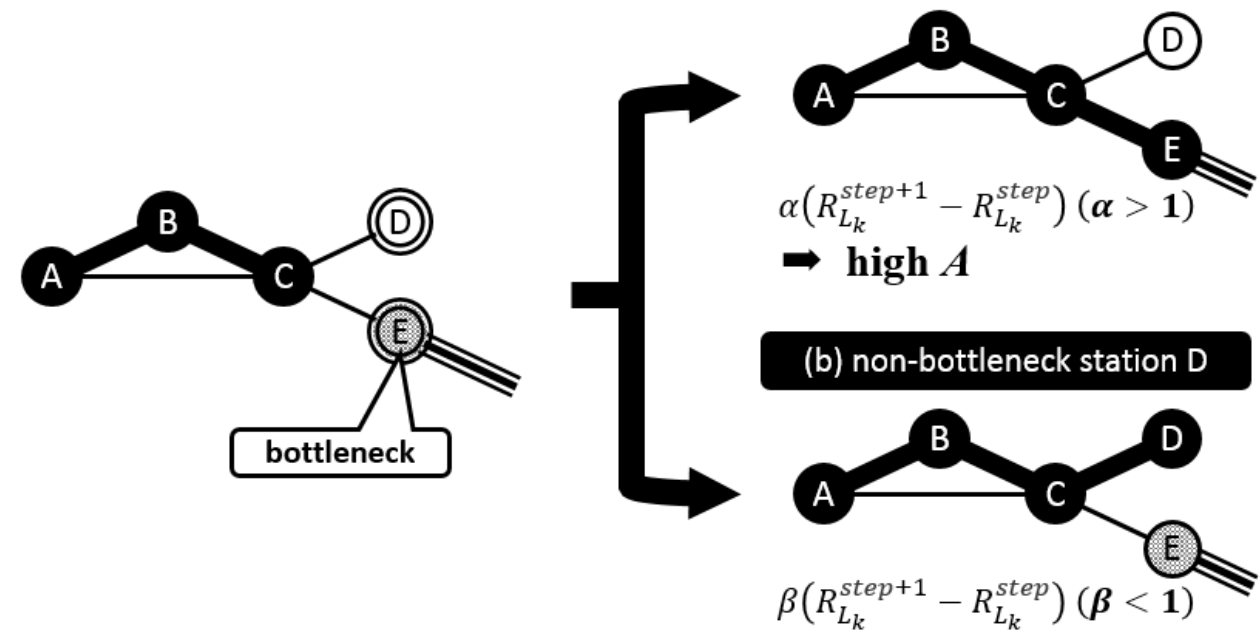

$\Rightarrow \operatorname{low} A$ 
Figure 5 Image of removing the station by the proposed method

removing

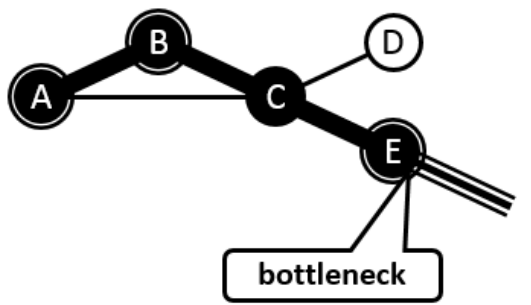

\section{(a) Bottleneck station $\mathrm{E}$}

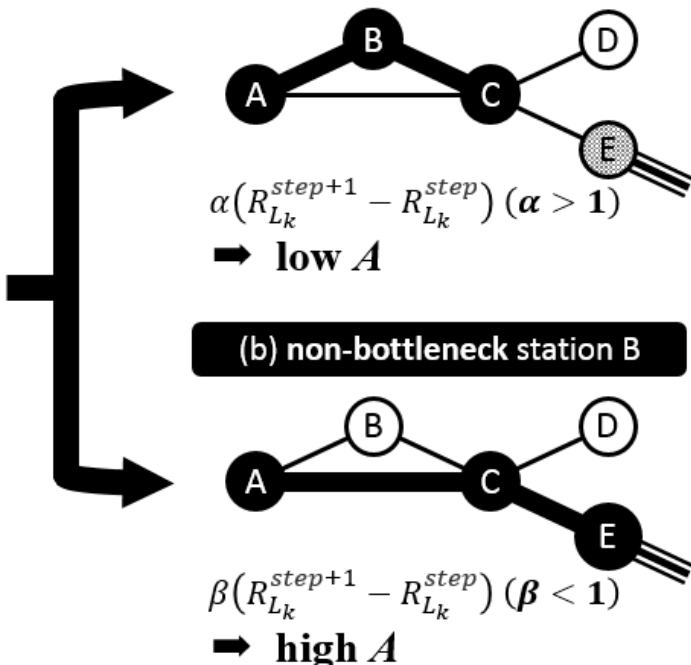

The images of an effect of this modification for adding/removing the station is shown in Figure 4 and Figure 5. In these images, the station E means to the bottleneck station. When adding the station, the bottleneck station E [Figure 4(a)] has a high possibility of being selected instead of the non-bottleneck station D [Figure 4(b)] because $\alpha$ $\left(R_{L_{k}}^{\text {step }+1}-R_{L_{k}}^{\text {step }}\right)$ tends to be larger than $\beta\left(R_{L_{k}}^{\text {step }+1}-R_{L_{k}}^{\text {step }}\right)$, resulting that the evaluation A adding the station $\mathrm{E}$ tends to be higher than the one adding the station $\mathrm{D}$ (Note that $R_{L_{k}}^{\text {step+1 }}$ in the former term is different from $R_{L_{k}}^{s t e p+1}$ in the latter term). Similarly, when removing the station, the non-bottleneck station B [Figure 5(b)] has a high possibility of being selected instead of the bottleneck station E [Figure 5(a)] because $\alpha\left(R_{L_{k}}^{\text {step }+1}-R_{L_{k}}^{\text {step }}\right)$ tends to be lower than $\beta\left(R_{L_{k}}^{\text {step+1 }}-R_{L_{k}}^{\text {step }}\right)$, resulting that the evaluation $\mathrm{A}$ removing the station $\mathrm{E}$ tends to be lower than the one removing the station $\mathrm{B}$. In this manner, the line non-having the bottleneck stations tends to include them, while the line having the bottleneck stations tends to exclude the non-bottleneck stations, in order to transfer many passengers at the bottleneck station by adding the new line or by reducing the length of the line for a high frequency of bus coming.

\subsection{Bus redistribution between lines (\#3.5 redistribute buses)}

Our proposed method has the unique mechanism to cope with the bottleneck situation by modifying the bus distribution. For this mechanism, the number of stranded persons in each line is calculated by 


$$
C_{L_{k}}=R_{L_{k}}-\left(\text { Capacity } \times B_{L_{k}}\right)
$$

where Capacity represents the capacity of a bus (which is fixed). When $C_{L_{k}}$ is positive value, all passengers in the line $L_{k}$ cannot be transferred, meaning that some passengers should wait at the station as stranded persons. When $C_{L_{k}}$ is negative value, on the other hand, all passengers in the line $L_{k}$ can be transferred, meaning that this line has an enough number of buses that can provide some buses to the other line.

As the mechanism of \#3.5 in Figure 2, the line having the smallest $C_{L_{k}}$ gives some buses to the line having the largest $C_{L_{k}}$ (Note that the line gives all buses to the line having the largest $C_{L_{k}}$ when the line is deleted as the unused one). Such a bus redistribution contributes to increasing the frequency of bus, which results in the situation where the waiting time of the passengers becomes shortly and the line can transport them quickly. This modification enables the lines to redistribute the number of buses properly under the condition where the total number of buses in the route network is fixed. Here, we define the redistribution number of buses between lines as RD.

Moreover, when deleting the unused line of \#3 in Figure 2, our method reuses the buses which are distributed to the unused line as redistribution to the other used line. For example, when the deleted line has three buses, after deleting these buses are given to the line having the largest $C_{L_{k}}$. It is because our method fixes the total number of buses in the route network to consider the disaster situation unlike the Majima's method, the unused buses should not be wasted.

\section{Problem description}

\subsection{Urban transport bench mark problem}

To investigate the effectiveness of the proposed method, we conducted the simulation using the Mandl's urban transport bench mark problem (Mandl, 1979). This problem consists of 15 stations, 21 roads (i.e., the link between stations). The physical infrastructure network is shown in Figure 6 where the circles indicate the stations, the lines indicate the roads, the circle numbers indicate the ID number of the station, and the number near the lines indicate the travelling time between the two stations by the bus.

Table 1 shows the OD table of the Mandl's urban transport bench mark problem, where the horizontal and vertical axes respectively indicates the origin and destination station ID numbers and the number in this table indicates the number of passengers per a day. Actually, all methods in our experiment optimise their route network based on the OD numbers per an hour, which are calculated by dividing the OD numbers per a day by 10 (i.e., as business hours in the bus company). According to Mandl (1979), the capacity of a bus is 50 passengers. 
Figure 6 Infrastructure network of Mandl's urban transport bench mark problem

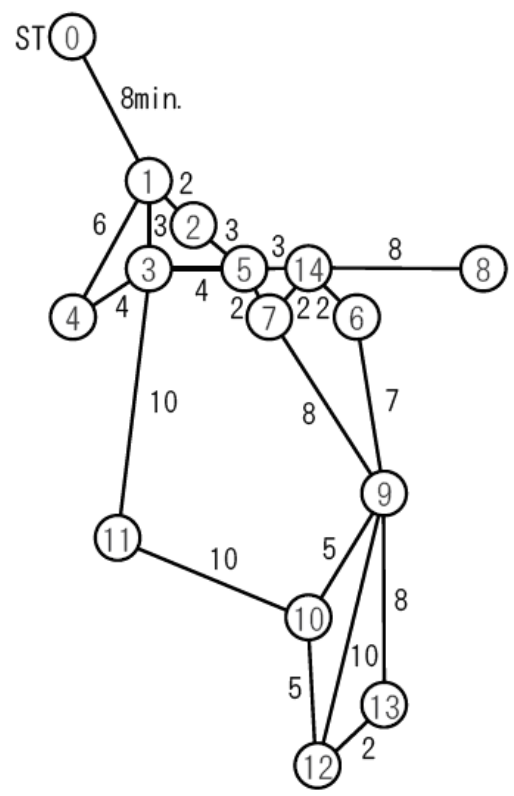

Table 1 OD table (persons/day)

\begin{tabular}{cccccccccccccccc}
\hline$S T$ & 0 & 1 & 2 & 3 & 4 & 5 & 6 & 7 & 8 & 9 & 10 & 11 & 12 & 13 & 14 \\
\hline 0 & 0 & 400 & 200 & 60 & 80 & 150 & 75 & 75 & 30 & 160 & 30 & 25 & 35 & 0 & 0 \\
1 & 400 & 0 & 50 & 120 & 20 & 180 & 90 & 90 & 15 & 130 & 20 & 10 & 10 & 5 & 0 \\
2 & 200 & 50 & 0 & 40 & 60 & 180 & 90 & 90 & 15 & 45 & 20 & 10 & 10 & 5 & 0 \\
3 & 60 & 120 & 40 & 0 & 50 & 100 & 50 & 50 & 15 & 240 & 40 & 25 & 10 & 5 & 0 \\
4 & 80 & 20 & 60 & 50 & 0 & 50 & 25 & 25 & 10 & 120 & 20 & 15 & 5 & 0 & 0 \\
5 & 150 & 180 & 180 & 100 & 50 & 0 & 100 & 100 & 30 & 880 & 60 & 15 & 15 & 10 & 0 \\
6 & 75 & 90 & 90 & 50 & 25 & 100 & 0 & 50 & 150 & 440 & 35 & 10 & 10 & 5 & 0 \\
7 & 75 & 90 & 90 & 50 & 25 & 100 & 50 & 0 & 15 & 440 & 35 & 10 & 10 & 5 & 0 \\
8 & 30 & 15 & 15 & 15 & 10 & 30 & 15 & 15 & 0 & 140 & 20 & 5 & 0 & 0 & 0 \\
9 & 160 & 130 & 45 & 240 & 120 & 880 & 440 & 440 & 140 & 0 & 600 & 250 & 500 & 200 & 0 \\
10 & 30 & 20 & 20 & 40 & 20 & 60 & 35 & 35 & 20 & 600 & 0 & 75 & 95 & 15 & 0 \\
11 & 25 & 10 & 10 & 25 & 15 & 15 & 10 & 10 & 5 & 250 & 75 & 0 & 70 & 0 & 0 \\
12 & 35 & 10 & 10 & 10 & 5 & 15 & 10 & 10 & 0 & 500 & 95 & 70 & 0 & 45 & 0 \\
13 & 0 & 5 & 5 & 5 & 0 & 10 & 5 & 5 & 0 & 200 & 15 & 0 & 45 & 0 & 0 \\
14 & 0 & 0 & 0 & 0 & 0 & 0 & 0 & 0 & 0 & 0 & 0 & 0 & 0 & 0 & 0 \\
\hline
\end{tabular}




\subsection{Evaluation criteria}

As evaluation criteria, all simulation results are evaluated by

1 the critical travelling time (hereafter we call it as CriticalTime) of all passengers

2 the number of the stranded passengers as the overflow persons.

CriticalTime means the travelling time that can be reduced by taking a bus efficiently, while the number of the stranded passengers means the number of persons who cannot return home by the current route network. The shorter CriticalTime is, the better route network is. CriticalTime is defined as follows.

$$
\text { CriticalTime }=T T T-T T T_{\min }
$$

where $T T T$ means the total travelling time of all passengers and $T T T_{\min }$ means the minimum travelling time which cannot be shortened physically (i.e., this time is at least required to move from the origin station to the destination station). Regarding TTT, it is calculated by as follows.

$$
T T T=\sum_{S_{i} \neq S_{j}}\left(\text { TravelTime }_{S_{i}, S_{j}}+\text { WaitTime }_{S_{i}, S_{j}}+\text { TransTime }_{S_{i}, S_{j}}\right)
$$

where TravelTime indicates the time of passengers who travel from $S_{i}$ to $S_{j}$ by riding the bus, WaitTime indicates the time of waiting the bus at the bus station before travelling from $S_{i}$ to $S_{j}$, and TransTime indicates the time of getting on and off the bus when travelling from $S_{i}$ to $S_{j}$.

\section{Case study}

\subsection{Overview of experiments}

Our experiments start by investigating the effectiveness of the proposed method by comparing with the conventional one in the typical disaster situation, then investigating it in the hard disaster situation and the comprehensive disaster situation from slight to extreme case. For this purpose, we conduct the three experiments shown in Table 2. Concretely, the typical disaster situation (i.e., the OD in one station becomes large due to many stranded persons) is addressed in the experiment 1 , the hard disaster situation (i.e., the OD in one station becomes larger than the typical situation) is tackled in the experiment 2, and the comprehensive disaster situation from easy to extreme case (i.e., the $\mathrm{OD}$ in one station becomes from small to quite large) is investigated in the experiment 3 .

Here, we compared two methods, Majima's one and proposed one because of the following reasons. As described in Section 1, other major route optimisation methods are hard to cope with disaster situations due to the fact that they optimise the whole network from scratch even though the passengers are confused in such a whole network optimisation. In comparison with these methods, the Majima's method can cope with disaster situations by modifying only routes which are damaged by the disaster. Moreover, the Majima's method can generate the best route network in comparison with 
other optimisation methods even in the usual situation (i.e., not disaster situation) (Majima et al., 2015).

Table 2 Overview of experiments

\begin{tabular}{ll}
\hline Experiments & \\
\hline Experiment 1 & Typical disaster situation \\
Experiment 2 & Hard disaster situation \\
Experiment 3 & Comprehensive disaster situation from slight to extreme case \\
\hline
\end{tabular}

\subsection{Experiment 1: typical disaster situation}

\subsubsection{Experimental cases}

The experiment 1 compares the following three cases in the typical disaster situation which means that many stranded persons occur while no such persons occur in the usual situation:

- Conventional method as a usual situation [the conventional method (usual) in short]

The route network is optimised by the conventional method [i.e., the Majima's method (Majima et al., 2015)] as a usual situation, i.e., this route network is optimised before the disaster occurs.

- Conventional method for a disaster situation [the conventional method (disaster) in short]

The route network is modified by the conventional method for the disaster situation from the one generated in the conventional method (usual). In this case, the number of buses is closed to the one in the conventional method (usual) as the approximated number of buses.

- Proposed method for a disaster situation [the proposed method (disaster) in short]

The route network is modified by the proposed method for the disaster situation from the one generated in the conventional method (usual). In this case, the number of buses is the same one in the conventional method (usual) as the fixed number of buses.

As shown in the above cases, the number of buses can be fixed by the proposed method (disaster), while the conventional method (disaster) adds the buses as many as possible. Since it is unfair to compare the results with the different number of buses, the number of buses in the route network among three cases is the same as or closed to the number of buses (i.e., 77) optimised by the conventional method (usual). Concretely, the number of buses optimised by the conventional method (disaster) is close to 77 by turning the weighting coefficient value of $\omega_{1}$ (note that the same number of buses cannot be found in any values of $\omega_{1}$, i.e., 75 buses is found as the closest to 77), and the one optimised by the proposed method (disaster) is fixed to 77 .

What should be noted here is that the total bus capacity is calculated by 3,850 (= 50 (number of passengers / bus) $* 77$ (buses)) but the actual total bus capacity is larger than 3,850 because some buses go and return in the bus line in one hour. In addition, the actual number of passengers in the route network is not easy to calculate 
from the OD table because they transfer between some lines to go to destination station. From this characteristic, we can only calculate the number of persons and the total bus capacity after generating the bus route network. For the difference between the usual and disaster situations, the passengers in the usual situation move according to the usual OD table shown in Table 1, while the passengers in the disaster situation move according to the disaster OD table which has the ten times number of passengers who depart from and arrived at the station no. 9. We select this station because the most number of passengers use this station and give a big influence to the current route network when the number of passengers in this station increases. Such an increase of the number of passengers is caused by an occurrence of the stranded persons in the disaster situation.

\subsubsection{Parameter setting}

Table 3 shows the weighting coefficient values for all cases. In the conventional method (usual) and the proposed method (disaster), the weighting coefficient values of $\omega_{1}$ and $\omega_{2}$ are set as the same value in the prior study (Majima et al., 2015) which reported that the parameters can derive the good results. In the conventional method (disaster), on the other hand, the weighting coefficient values of $\omega_{1}$ and $\omega_{2}$ are set so that the number of buses in the route network becomes to be close to the one (i.e., 77) in the route network as usual situations. The number of the buses is determined by the weighting coefficient values of $\omega_{1}$ and $\omega_{2}$ in Table 3. As the parameters for the proposed method (disaster), $\omega_{3}$, $\alpha, \beta$, and $R D$ (redistribution number of buses between lines) are set as shown in Table 4 . Here, $R D$ is determined according to the pre-experiment conducted to find the best $R D$.

Table 3 Parameter setting of the weighting coefficient values $\left(\omega_{1}\right.$ and $\left.\omega_{2}\right)$

\begin{tabular}{lllc}
\hline Cases & $\omega_{1}$ & $\omega_{2}$ & Number of buses \\
\hline Conventional method (usual) & 0.7 & 1.0 & 77 \\
Conventional method (disaster) & 3.7 & 1.0 & 75 \\
Proposed method (disaster) & 0.7 & 1.0 & 77 \\
\hline
\end{tabular}

Table 4 Parameter setting of the proposed method (disaster)

\begin{tabular}{lccc}
\hline$\omega_{3}$ & $\alpha$ & $\beta$ & $R D$ \\
\hline 2.0 & 1.5 & 0.5 & 3 \\
\hline
\end{tabular}

\subsubsection{Results}

Figure 7(a) shows the total travelling time (TTT) and the critical travelling time (CriticalTime) of all passengers, where the left vertical, right vertical, and horizontal axes respectively indicate $T T T$, CriticalTime, and three cases [i.e., the conventional method (usual), the conventional method (disaster), and the proposed method (disaster)]. Figure 7(b), on the other hand, shows the number of the stranded passengers in three cases, where the vertical and horizontal axes respectively indicate the number of the stranded passengers and three cases. Since the results in all three cases change better and worse through the evolutionary process, we select one of them which shows the shortest CriticalTime meaning the most superior route network. 
Figure 7 Comparison of three cases in experiment 1, (a) critical travelling time (CriticalTime) and total travelling time (TTT) (b) number of stranded passengers

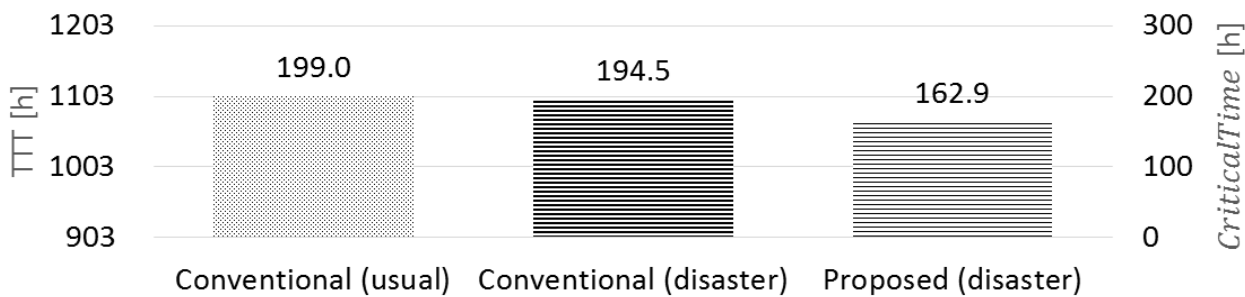

(a)

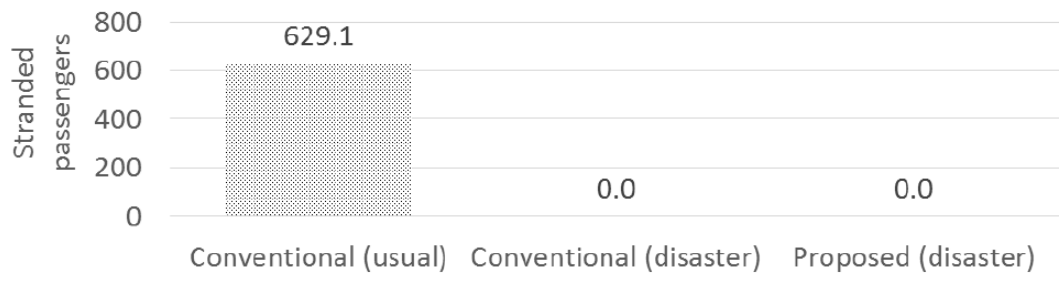

(b)

From Figure 7(a), both CriticalTime and TTT in the proposed method (disaster) are shorter than those in other cases. In particular, the proposed method (disaster) can reduce approximately $18 \%$ of CriticalTime in comparison with the conventional methods. From Figure 7(b), on the other hand, 629.1 passengers become the stranded persons in the conventional method (usual), while all passengers can return their home in the conventional method (disaster) and proposed method (disaster). These results suggest that the proposed method (disaster) have a potential of reducing both CriticalTime and TTT while keeping none of stranded persons.

Since the result of the conventional method (disaster) is based on 75 buses which is shorter than 77, we also investigated how the result changes in the case of the larger number of buses than 75 , i.e., 81 is the next nearest number of 77 . In the case of 81 buses, however, the result showed the longer TTT and CriticalTime (196.67) than those optimised by conventional method with 75 buses (194.5) and by the proposed one with 77 buses (162.9). This means that an addition of more number of buses does not always contribute to reducing CriticalTime because it is depend on not only the number of buses but also the route networks which consists of some lines.

\subsubsection{Discussion}

To explore the reasons why we obtained the above results in the experiment 1 , this subsubsection analyses the route networks optimised by the conventional method (usual), the conventional method (disaster), and the proposed method (disaster) as shown in Figure 8. In this figure, the arrows indicate the lines, and the very thick line in particular indicates the road in which the bottleneck occurs. The circles indicate the station and the black-filled circle in particular indicates the station 9 which OD is multiplied. Note that the roads between stations $\{6,7$, or 10$\}$ and 9 have a possibility of becoming the bottleneck roads due to an increase of the number of passengers in the station 9 , and the road between stations 7 and 9 becomes the bottleneck road in this experiment. 
Figure 8 Route networks of the conventional and proposed methods

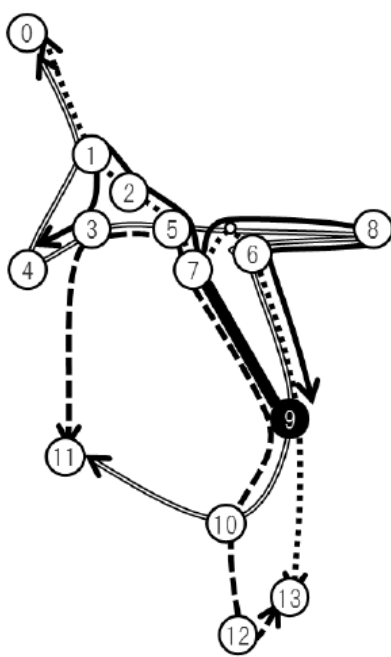

Conventional (usual)

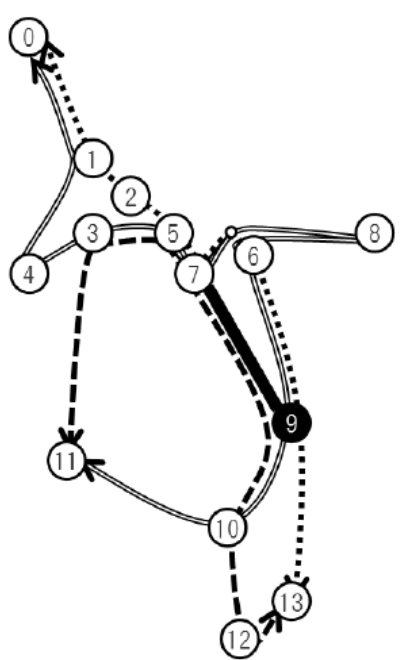

Conventional (disaster)

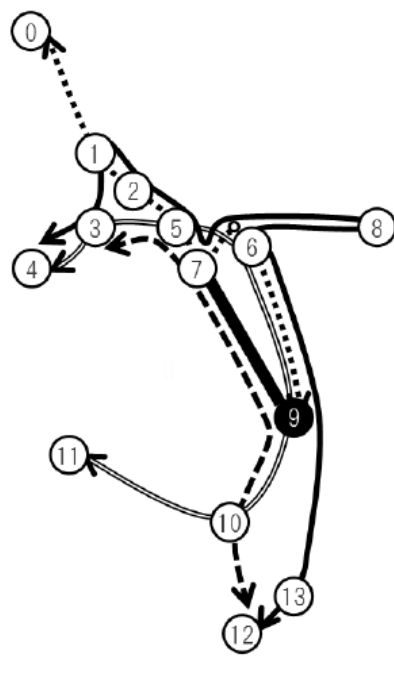

Proposed (disaster)

When focusing on the conventional method (usual), it optimises the route network without considering the disaster situation, which means that the evolved route network is generally hard to be applied in the disaster situation. To understand this meaning, let's analyse the double line (composed of the stations $0,1,4,3,5,8,6,9$, 10, and 11, i.e., no station 7) evolved by the conventional method (usual) and the double line (composed of the stations $0,1,4,3,5,7,8,6,9,10$, and 11, i.e., an inclusion of the station 7) evolved by the conventional (disaster). Since the route network optimised by the conventional methods (usual) does not include the station 7, the stranded persons appear due to an excess of the number of passengers at the station 7 which comes from the station 9 . In contrast, since the route network optimised by the conventional methods (disaster) includes the station 7 , none of the stranded persons appears in even in such a situation. This derives the result shown in Figure 7(b).

By comparing the route networks optimised by the conventional method (disaster) and the proposed method (disaster), the lines including the bottleneck road between 7 and 9 are evolved by both methods, but the length of the dashed line (composed of 3, 5, 7, 9, 10 , and 12) in the conventional method (disaster) is two bus stops shorter than that of the dashed line (composed of 11, 3, 5, 7, 9, 10, 12, and 13) in the conventional method (disaster). This suggests that the waiting time of the passengers in the line evolved by the proposed method (disaster) becomes shorter than that in the line evolved by the conventional method (disaster). This derives the result shown in Figure 7(a) suggesting that CriticalTime of the proposed method (disaster) becomes shorter than that of the conventional method (disaster).

From these analyses, the proposed method (disaster) has a potential of reducing CriticalTime while keeping none of stranded persons. 


\subsection{Experiment 2: hard disaster situation}

\subsubsection{Experimental cases}

Since the experiment 1 only investigated a certain disaster situation, the experiment 2 compares the following three cases (all of which are the same as the ones in the experiment 1) to investigate whether the proposed method (disaster) can cope with a hard disaster situation, which means that more number of the stranded persons occur in comparison with the situation in the experiment 1 . Concretely, the number of passengers becomes 10 and higher (i.e., 11, 12, 13, and 14) times number of passengers who depart from and arrived at the station no. 9 .

- conventional method as a usual situation [the conventional method (usual) in short]

- conventional method for a disaster situation [the conventional method (disaster) in short]

- proposed method for a disaster situation [the proposed method (disaster) in short].

\subsubsection{Parameter setting}

The weighting coefficient values of $\omega_{1}$ and $\omega_{2}$ except for the conventional method (disaster) and the values of $\omega_{3}, \alpha, \beta$, and $R D$ (redistribution number of buses between lines) are set as the same ones in the experiment 1 . In the conventional method (disaster), in particular, the weighting coefficient values of $\omega_{1}$ and $\omega_{2}$ are set as shown in Table 5 so that the number of buses in the route network becomes to be close to the one in the route network as usual situations. The number of the buses is determined by the weighting coefficient values of $\omega_{1}$ and $\omega_{2}$ in Table 5. Note that these numbers are hard to be kept around 77 as the multiplied number of passengers increases (i.e., as OD has more than $11,12,13$, and, 14 times number of passengers who depart from and arrived at the station no. 9).

Table 5 Parameter setting of the weighting coefficient values $\left(\omega_{1}\right.$ and $\left.\omega_{2}\right)$ in conventional method (disaster)

\begin{tabular}{lllc}
\hline Conventional method (disaster) & $\omega_{1}$ & $\omega_{2}$ & Number of buses \\
\hline 10 times number of passengers & 3.7 & 1.0 & 75 \\
11 times number of passengers & 5.9 & 1.0 & 79 \\
12 times number of passengers & 5.4 & 1.0 & 86 \\
13 times number of passengers & 9.8 & 1.0 & 92 \\
14 times number of passengers & 9.8 & 1.0 & 99 \\
\hline
\end{tabular}

\subsubsection{Results}

Figure 9(a) shows the critical traveling time (CriticalTime) of all passengers, where the vertical and horizontal axes respectively indicate CriticalTiime and the multiplied number of passengers. In this figure, the solid, dashed, and dotted line indicates CriticalTime of three cases [i.e., the conventional method (usual), the conventional method (disaster), and the proposed method (disaster)]. Figure 9(b), on the other hand, shows the number of the stranded passengers, where the vertical and horizontal axes respectively indicate the 
number of the stranded passengers and the multiplied number of passengers. In this figure, the bar filled by the thick lines indicates the results of the conventional method (usual) and the bar filled by the grey with the white dots indicates the results of the proposed method (disaster). Since the results in all three cases change better and worse through the evolutionary process as the same as the experiment 1 , we select one of them which shows the shortest CriticalTime, meaning the most superior route network.

Figure 9 Comparison of three cases in experiment 2, (a) critical travelling time (CriticalTime) (b) number of stranded passengers

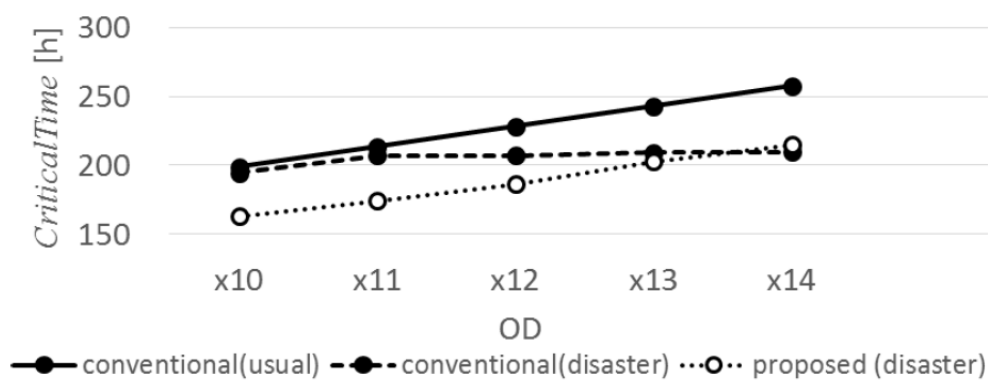

(a)

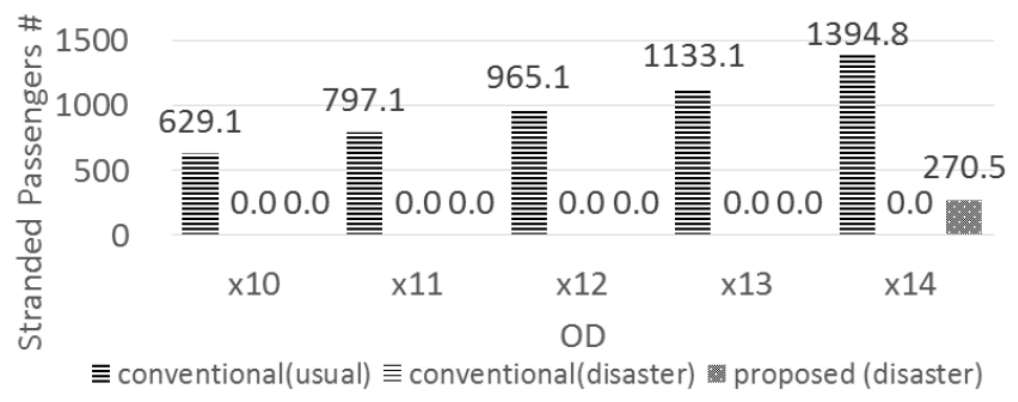

(b)

From Figure 9(a), CriticalTime in the proposed method (disaster) is shortest in comparison with the both conventional methods (usual) and (disaster) except for the situation of the 14 times number of passengers. From Figure 9(b), none of the stranded number of passenger appears in both the conventional method (disaster) and the proposed method (disaster) except for the situation of the 14 times number of passengers. On the other hand, both CriticalTime and the stranded number of passengers increase in the conventional method (usual) as the number of passengers increases at the station. These results suggest that the conventional method (usual) cannot be applied in the disaster situation because this method cannot prevent from an occurrence of the stranded persons.

\subsubsection{Discussion}

The above result shown in Figure 9 seems that both the conventional method (disaster) and the proposed method (disaster) work well in hard disaster situations. In order to clarify this implication, we investigate the number of buses introduced in the route network shown in Figure 10 where the vertical and horizontal axes respectively indicate 
the number of buses and the multiplied number of passengers. In this figure, the bar filled by the thick lines indicates the results of the conventional method (usual), the bar filled by the thin lines indicates the results of the conventional method (disaster), and the bar filled by the gray with the white dots indicates the results of the proposed method (disaster). Note that the thick dotted line indicates the number of buses (i.e., 77) optimised by the conventional method (usual).

Figure 10 Number of buses in experiment 2

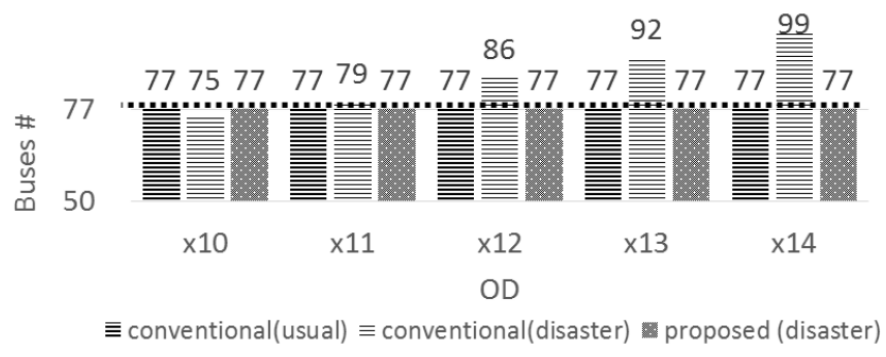

From the result shown in Figure 10, the conventional method (disaster) requires many buses more than 77 as the number of passengers increases, while the proposed method (disaster) can keep the 77 buses as well as the conventional method (usual). This suggests that the proposed method (disaster) is the only method that has a potential of coping with the hard disaster situations by not only keeping the number of buses as the same as its total number but also reducing the CriticalTime without stranded persons except for the situation of the 14 times number of passengers. Note that the stranded persons appear in the only exception situation (i.e., the 14 times number of passengers at the station 9) because the total number of passengers is larger than the total capacity of the buses, i.e., all passengers cannot be carried by any methods under the current number of buses. This suggests that an occurrence of the stranded persons in the proposed method (disaster) is not significant problem.

\subsection{Experiment 3: comprehensive disaster situation}

\subsubsection{Experimental case}

Since the experiments 1 and 2 suggests that the proposed method (disaster) can cope with the situations from the typical to hard disaster, the experiment 3 investigates its capability against the disaster situations comprehensively. Concretely, in the harder situation than the experiment 2, we do not know how the result changes when the total number of passengers exceeds the capacity of the total buses. If CriticalTime increases drastically in the harder disaster situations, our proposed method will not perhaps adjust it well. For this purpose, the experiment 3 investigates how the proposed method (disaster) copes with the different situations where the total number of passengers does not exceed or exceeds the capacity of the total buses. Concretely, this experiment addresses the situation which has a 5,10,15, and 20 times number of passengers who depart from and arrived at the station no. 9. Note that the total number of passengers does not exceed the capacity of the total buses in the case of 5 and 10 times number of passengers, while the 
total number of passengers exceeds the capacity of the total buses in the case of 15 and 20 times number of passengers.

\subsubsection{Parameter setting}

The weighting coefficient values of $\omega_{1}$ and $\omega_{2}$ and the values of $\omega_{3}, \alpha, \beta$, and $R D$ (redistribution number of buses between lines) are set as the same ones in the experiments 1 and 2 .

\subsubsection{Results}

Figure 11(a) shows the critical travelling time (CriticalTime) of all passengers, where the vertical and horizontal axes respectively indicate CriticalTime and the multiplied number of passengers. In this figure, the dashed line indicates CriticalTime of the proposed method (disaster). Figure 11(b), on the other hand, shows the number of the stranded passengers, where the vertical and horizontal axes respectively indicate the number of the stranded passengers and the multiplied number of passengers. In this figure, the bar filled by the gray with the white dots indicates the results of the proposed method (disaster). Since the results in this case change better and worse through the evolutionary process as the same as the experiments 1 and 2, we select one of them which shows the shortest CriticalTime meaning the most superior route network.

Figure 11 Disaster capability of the proposed method in experiment 3, (a) critical travelling time (CriticalTime) (b) number of stranded passengers

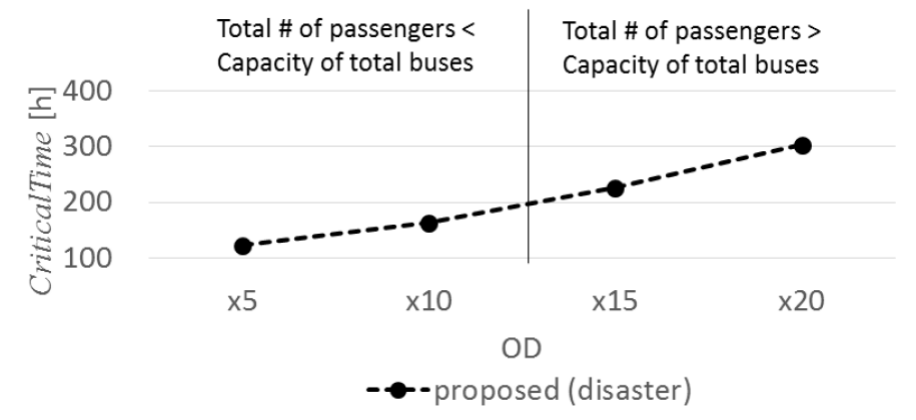

(a)

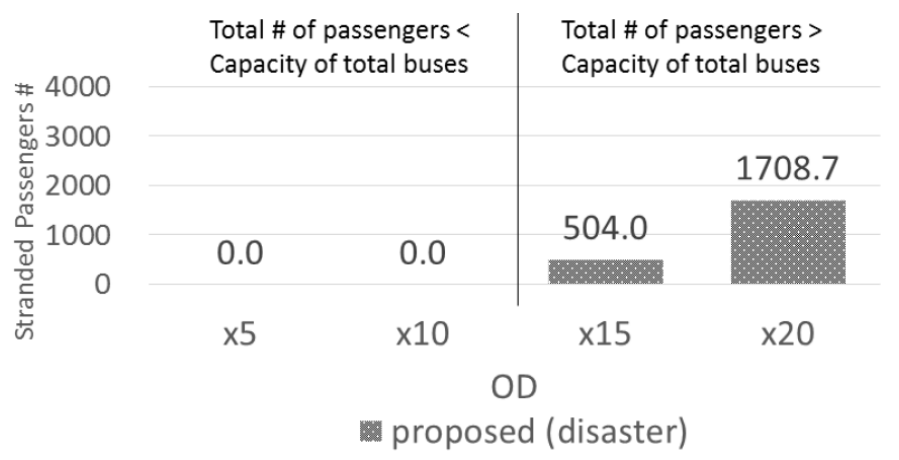

(b) 
From Figure 11(a), CriticalTime in the proposed method (disaster) increases as the number of passengers increases at the station. This tendency does not drastically change in the situations where the total number of passengers does not exceed or exceeds the capacity of the total buses. This is a very important capability of the proposed method (disaster) because CriticalTime does not drastically increase even when the total number of passengers exceeds the capacity of the total buses, which can be occurred in terrible disasters. From Figure 11(b), on the other hand, the number of stranded persons in the proposed method (disaster) increases as the number of passengers increases. In particular, none of the stranded persons appears when the total number of passengers does not exceed the capacity of the total buses, while those persons appear when the total number of passengers exceeds the capacity of the total buses. This is natural result because the total number of buses (i.e., 77) is fixed even then the total number of passengers exceeds the capacity of the total buses. Note that this is not a fault of the proposed method (disaster), i.e., none of methods cannot transport the passengers when the total number of passengers exceeds the capacity of the total buses.

\subsubsection{Discussion}

To investigate the result obtained in the experiment 3 , this subsubsection analyses the route networks optimised by the proposed method (disaster) in different disaster situations as shown in Figure 12. Concretely, the left figure in Figure 12 indicates the evolved route network against the situation where the ten times number of passengers appear in the station 9 meaning that the total number of passengers does not exceed the capacity of the total buses (hereafter we call this situation as the non-exceeded case), while the right figure in Figure 12 indicates the evolved route network against the situation where the 20 times number of passengers appear in the station 9 meaning that the total number of passengers exceed the capacity of the total buses (hereafter we call this situation as the exceeded case). Note that the left figure in Figure 12 is the same as the right figure in Figure 8. In this figure, the arrows (including the very thick line) and the circle (including the black-filled one) have the same meaning of Figure 8.

Through a comparison of the route networks optimised in the non-exceeded and exceeded cases respectively shown in Figure 12, we focus on the solid line which includes the station 9. From the route networks, the length of the solid line (composed of $4,3,1,2,5,7,8,6$, and 9) in the exceeded case (shown in the right figure of Figure 12) is two bus stops shorter than that of the solid line (composed of $4,3,1,2,5,7,8,6,9,13$, and 12 ) in the non-exceeded case (shown in the left figure of Figure 12).

This suggests that the proposed method tries to minimise the waiting time of the passengers even when the total number of passengers exceed the capacity of the total buses. This capability of the proposed method (disaster) contributes to not drastically increasing CriticalTime even in the exceeded case shown in Figure 11(a). From the above analysis, the proposed method (disaster) has a wide capability from the non-exceeded case to the exceeded case. 
Figure 12 Route networks of the proposed method in different ODs $(\times 10$ and $\times 20)$

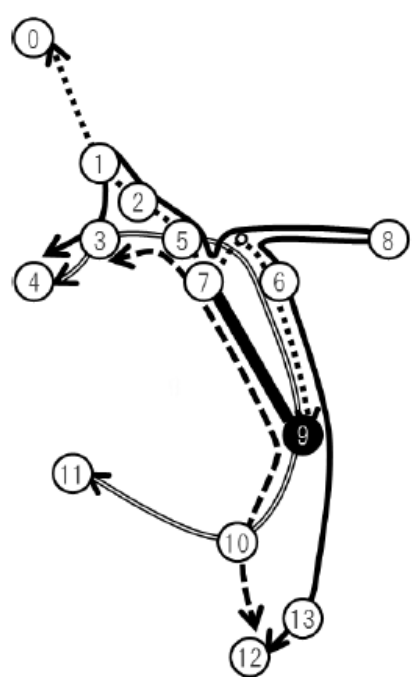

Proposed (disaster)

[OD x10]

Total \# of passengers < Capacity of total buses

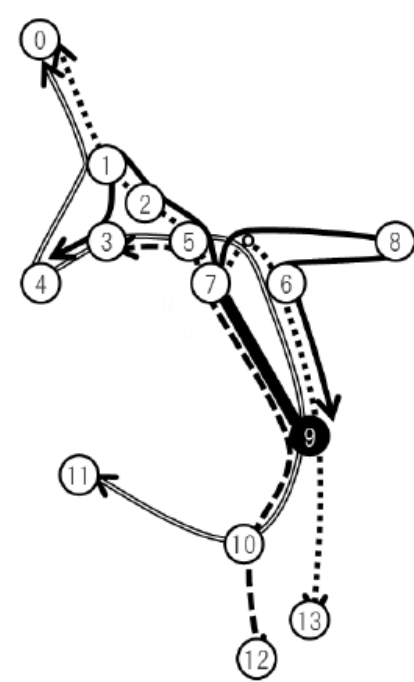

Proposed (disaster)

[OD $\times 20]$

Total \# of passengers >

Capacity of total buses

\section{Conclusions}

This paper focused on the passenger traffic bottlenecks occurred in the bus route network in disaster situations and proposed the multi-agent-based bus route network optimisation method to solve such bottlenecks by generating the networks which can effectively transport many stranded persons including ones who wait around the station as the passenger traffic bottlenecks. For this purpose, the proposed method modifies the bus route networks generated as usual situations to suitably generate many bus lines and redistribute the buses among the bus lines according to the number of passengers. Such an approach by the proposed method contributes to resolving the passenger traffic bottlenecks and transporting stranded passengers quickly. Through the simulations on the Mandl's urban transport benchmark problem, we have revealed the following implications:

1 From the experiment 1, the proposed method can optimise the bus route network which is suitable for the passenger traffic bottlenecks. Concretely, our method can reduce approximately $18 \%$ of the passengers' travelling time without stranded persons in comparison with the conventional method.

2 From the experiment 2, the proposed method optimises the bus route network which can cope with the hard disaster situations (where the number of passengers increases) without an inclusion of the additional buses, while the conventional method requires many buses when the number of passengers increases. 
3 From the experiment 3, the proposed method has the capability of decreasing a risk of the bottlenecks by concentrating on a modification of the lines having the bottleneck stations through the bus route network optimisation.

Concretely, the proposed method tries to optimise the route network even when the total number of passengers exceeds the capacity of the total buses.

These implications suggest that the proposed method gives the great potential of transporting many stranded persons in the disasters situations. What should be noticed here is that these results have only been obtained from one test-bed problem, i.e., the Mandl's urban transport benchmark problem. Therefore, further careful qualifications and justifications, such as an analysis of results using other but route networks, are needed to generalise our results. Such important directions must be pursued in the near future in addition to the following research:

1 addressing the situation where two or more bottlenecks occur simultaneously

2 introducing the persons who walk from the station to their home

3 exploring the automated determination method for the redistribution number of buses.

\section{References}

Baaj, M.H. and Mahmassani, H.S. (1995) 'Hybrid route generation heuristic algorithm for the design of transit networks', Transportation Research, Vol. 3, No. 1, pp.31-50.

Cabinet Office and the Central Disaster Prevention Council (2011) Annual Report Summary about Metropolitan Area Maintenance (in Japanese).

Central Disaster Management Council (2005) About Damage Estimation Result Pertaining to Tokyo Inland Earthquakes, CAO (in Japanese).

Chakroborty, P. and Wivedi, T. (2002) 'Optimal route network design for transit systems using genetic algorithms', Engineering Optimization, Vol. 34, No. 1, pp.83-100.

Fan, W. and Machemehl, R.B. (2006) 'Using a simulated annealing algorithm to solve the transit route network design problem', Journal of Transportation Engineering, Vol. 132, No. 2, pp.122-132.

Gen, M., Cheng, R. and Lin, L. (2008) Network Models and Optimization: Multi-Objective Genetic Algorithm Approach, Springer-Verlag, London.

Kitagawa, H., Sato, K. and Takadama, K. (2014) 'Robust bus route optimization to destruction of roads', Journal of Information Processing, Vol. 2, No. 22, pp.235-242.

Majima, T., Takadama, K., Watanabe, D. and Katsuhara, M. (2015) 'Characteristic of passenger's route selection and generation of public transport network', SICE Journal of Control, Measurement, and System Integration (JCMSI), Vol. 8, No. 1, pp.67-73.

Mandl, C. (1979) 'Evaluation and optimization of urban public transportation networks', The 3rd European Congress on Operation Research, pp.396-404.

Morimoto, S., Jimba, T., Kitagawa, H., Takadama, K., Majima, T., Watanabe, D. and Katsuhara, M. (2014) 'Multi-agent based bus route optimization for restricting passenger traffic bottlenecks in disaster situations', The 18th Asia Pacific Symposium on Intelligent and Evolutionary Systems, Springer, Vol. 2, pp.415-428. 
Ngamchai, S. and Lovell, D.J. (2003) 'Optimal time transfer in bus transit route network design using a genetic algorithm', Journal of Transportation Engineering, Vol. 129, No. 5, pp.510-521.

Zhao, F. and Ubaka, I. (2004) 'Transit network optimization of minimizing transfers and optimizing route directness', Journal of Public Transportation, Vol. 7, No. 1, pp.67-82.

Zhao, F. and Zeng, X. (2007) 'Optimization of user and operator cost for large-scale transit network', Journal of Transportation Engineering, Vol. 133, No. 4, pp.240-251. 